\title{
Gestion efficace des admissions ou monstre bureaucratique?
}

\author{
Jürg Schlup \\ Dr méd., président de la FMH
}

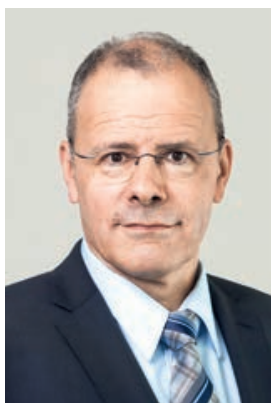

En matière d'admission, un seul point fait l'unanimité dans le débat actuel: la nécessité d'une régulation qui répond aux besoins et aux exigences de qualité de notre système de santé. Mais sur la manière d'y parvenir, les avis sont malheureusement partagés.

Depuis des années, le corps médical défend une solution simple et efficace: trois années d'activité hospitalière en Suisse ainsi qu'une attestation de compétence linguistique comme prérequis à une admission (voir ci-dessous). A contrario, le Conseil fédéral a présenté en mai 2017 un projet de loi (18.047) prévoyant une régulation particulièrement complexe [1]. Depuis les dernières modifications apportées au projet par le Conseil national en décembre 2018, il n'y a plus de doute possible: «Un nouveau monstre bureaucratique nous menace» [2], selon la formulation de Helsana.

\section{La moindre modification du taux d'activité des} 19331 médecins ambulatoires est-elle censée occuper les administrations cantonales à l'avenir?

C'est les administrations cantonales qui devraient gérer une grande partie de cette inflation bureaucratique. Il faudrait non seulement une planification détaillée de l'offre; la régulation des admissions en fonction du nombre défini de médecins exigerait de l'administration un contrôle régulier du taux d'occupation de tous les médecins. Ainsi, les 19331 médecins exerçant en Suisse [3] dans le secteur ambulatoire seraient, à l'avenir, tenus de communiquer au canton toute modification de leur taux d'activité, ou de la lui soumettre pour approbation. Une adaptation de la charge de travail des médecins indépendants souhaitant assumer, à long terme ou pas, des missions de médecin scolaire, de médecin-conseil ou d'expert, ou remplacer un confrère, ne pourra plus s'effectuer à l'amiable, mais devra passer par les méandres de l'administration cantonale.

L'ironie est que les cantons, malgré la lourde charge du contrôle, n'auraient pas plus, mais moins de marge de manœuvre. En effet, le projet de suppression de la clause du besoin à l'art. 55a LAMal et l'obligation de conditions d'admission à l'art. 37 LAMal leur ôteraient toute réactivité en cas de sous-approvisionnement Avec la nouvelle disposition prévue à l'art. 55a qui impose un gel des admissions pour toutes les spécialités présentant une hausse des coûts «supérieure à la moyenne», il est déjà statistiquement prévisible que, chaque année, les cantons ne pourront pas octroyer d'admission à la moitié au moins des disciplines, quel que soit le montant absolu de cette hausse, et surtout quelle que soit la demande réelle.

L'exigence politique "l'ambulatoire avant le stationnaire» deviendrait de ce fait aberrante: le transfert des coûts dû à un nombre accru de traitements ambulatoires se traduirait par le gel des admissions pour les spécialités concernées. Ce projet sous-tend ainsi une politique structurelle favorable au domaine hospitalier et accentue les conflits d'intérêts des cantons. La réglementation prévue, d'une grande complexité et associée à beaucoup de bureaucratie, ne laisse même pas espérer d'effets souhaitables. Alors qu'il serait plus simple et efficace de renforcer et de pérenniser les critères de qualité selon l'art. 55a LAMal, ce qui a été éprouvé depuis 2013. Si, premièrement, les trois années de pratique désormais exigées dans un établissement de formation postgraduée suisse reconnu étaient en plus effectuées dans la discipline médicale concernée par la demande d'admission, les cantons disposeraient d'un moyen de régulation simple permettant de garantir un mélange de spécialistes bien ajusté. Si, deu-

\section{L'exigence politique «ambulatoire avant} stationnaire" devient aberrante si plus de traitements ambulatoires entraînent le gel des admissions dans ce secteur.

xièmement, on exige en plus une attestation de la compétence linguistique nécessaire, l'admission s'en trouverait consolidée et la sécurité des patients améliorée. Cette solution simple et efficace suivrait non seulement la volonté du Conseil des Etats qui vient de voter un frein à la réglementation (16.3360), mais répondrait également à l'exigence d'un système de santé durable. 